\title{
openheart Smoking intensity and duration is associated with cardiac structure and function: the ECHOcardiographic Study of Hispanics/Latinos
}

\author{
J Adam Leigh, ${ }^{1}$ Robert C Kaplan, ${ }^{2}$ Katrina Swett, ${ }^{1}$ \\ Pelbreton Balfour, ${ }^{3}$ Mayank M Kansal, ${ }^{4}$ Gregory A Talavera ${ }^{5}$ Krista Perreira, ${ }^{6}$ \\ Michael J Blaha, ${ }^{7}$ Emelia J Benjamin, ${ }^{8}$ Rosemarie Robertson, ${ }^{9}$ Aruni Bhartnagar, ${ }^{10}$ \\ Carlos J Rodriguez ${ }^{1}$
}

\begin{abstract}
- Additional material is published online only. To view please visit the journal online (http://dx.doi.org/10.1136/ openhrt-2017-000614)
\end{abstract}

To cite: Leigh JA, Kaplan RC, Swett $\mathrm{K}$, et al. Smoking intensity and duration is associated with cardiac structure and function: the ECHOcardiographic Study of Hispanics/Latinos. Open Heart 2017;4:e000614. doi:10.1136/ openhrt-2017-000614

Received 21 February 2017 Revised 7 March 2017 Accepted 29 March 2017

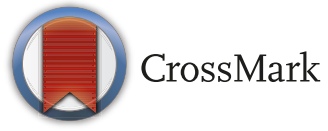

For numbered affiliations see end of article.

Correspondence to Dr Carlos J Rodriguez; crodrigu@wakehealth.edu

\section{ABSTRACT}

Objective Cardiovascular disease is the leading cause of death in smokers and this relationship is complicated by the multiplicity of cardiovascular effects of smoking. However, the relationship between intensity and duration of cigarette smoking and echocardiographic measures of right and left ventricular structure and function has been poorly studied.

Methods We examined ECHO-SOL (Echocardiographic Study of Hispanics/Latinos) participants, a subset of the Hispanic Community Health Study/Study of Latinos. Participants were administered a detailed tobacco exposure questionnaire and a comprehensive echocardiography exam. Multivariable linear regression models (adjusted for age, sex, obesity, hypertension and diabetes statuses) were performed using sampling weights. Statistical significance was defined at $p \leq 0.01$. Results There were 1818 ECH0-SOL participants $(57.4 \%$ women, mean age 56.4 years). Among current smokers $(\mathrm{n}=304)$, increased duration of smoking, as measured by a younger age of smoking initiation, was significantly associated with higher mean left ventricular mass (LVM) and lower right ventricular (RV) function (lower right ventricular stroke volumes). More cigarettes smoked per day was significantly associated with higher mean LVM, worse diastolic function (higher E/e' ratio), worse LV geometry (increased relative wall thickness) and worse RV function (decreasing right ventricular stroke volume). Among current smokers, higher mean lifetime pack-years (a combined measure of smoking intensity and duration) was associated with higher LVM, worse LV geometry, worse diastolic function, greater RV dilatation and worse RV function.

Conclusions There is a dose-response relationship between intensity and duration of cigarette tobacco smoking with unfavourable changes of multiple measures of right-sided and left-sided cardiac structure and function.

\section{INTRODUCTION}

Cigarette smoking is a well-known and preventable risk factor for atherosclerotic cardiovascular disease. ${ }^{1}$ The American

\section{KEY MESSAGES}

What is already known about this subject? Cardiovascular disease is the leading cause of death in smokers and this relationship is complicated by the multiplicity of cardiovascular effects of smoking. Traditionally, pack-years has been a standard clinical history assessment of total tobacco exposure.

What does this study add?

With this study, there is evidence that there are cardiac abnormalities of structure and function associated with the intensity and duration of cigarette use in a relatively young cohort which could serve as important data for clinicians to use when counselling their patients on tobacco cessation.

How might this impact on clinical practice? Cigarette smoking exposure may impact the development of cardiomyopathy in a direct, continuous dose-dependent fashion and thus may contribute to the heart failure epidemic. The potential contribution of tobacco exposure to cardiomyopathy development deserves further study and public health vigilance.

Heart Association has identified abstaining from or quitting cigarette smoking as a major component of cardiovascular health. ${ }^{2}$ Over the past few decades, the rate of cigarette smoking in adults has decreased in the United States but still remains significant at $16.8 \% .^{3}$ Whereas the decreasing rates are encouraging, there are approximately 40 million adults still smoking cigarettes. In 2014, $10.7 \%$ of Hispanics/Latinos were current smokers and an additional $16.5 \%$ were former smokers. ${ }^{4}$

The relationship between cardiovascular disease and tobacco use is complicated by the multiplicity of the cardiovascular effects that are a result of smoking. In addition 


\begin{tabular}{|c|c|c|c|c|}
\hline Variable & Total & Never & Former & Current \\
\hline Age, years & $56.4 \pm 0.4$ & $56.1(0.5)$ & $58(0.5)$ & $55(0.6)$ \\
\hline Female, $\%$ & $57.4(1187)$ & 68.1(786) & $42.4(238)$ & $45.0(163)$ \\
\hline Body mass index, mean $\left(\mathrm{kg} / \mathrm{m}^{2}\right)$ & $30.1 \pm 0.2$ & $30.6(0.3)$ & $30.0(0.3)$ & $29(0.4)$ \\
\hline Fasting glucose & $106 \pm 1.1$ & $105(1.4)$ & $109(1.8)$ & $108(2.3)$ \\
\hline Heart rate (bpm) & $66 \pm 0.3$ & $66(0.4)$ & $65(0.6)$ & $66(0.7)$ \\
\hline Hypertension, \% & $50(861)$ & $497(49.4)$ & $227(52.9)$ & $137(47.9)$ \\
\hline Systolic BP (mm Hg) & $136 \pm 0.6$ & $137(0.9)$ & $137(1.2)$ & $134(1.3)$ \\
\hline Diastolic BP (mm Hg) & $78 \pm 0.4$ & $78(0.5)$ & $78(0.7)$ & $78(0.9)$ \\
\hline Total cholesterol (mg/dL) & $209 \pm 1.4$ & $210(1.7)$ & $210(3.3)$ & $201(3.2)$ \\
\hline Chronic kidney disease, \% & $6.4(106)$ & $6.7(65)$ & $6.3(24)$ & $5.8(17)$ \\
\hline Smoking status, $\%$ & & $56.7(1069)$ & $25.7(443)$ & $17.6(304)$ \\
\hline Current non-daily smokers & & & & $19.1(66)$ \\
\hline Current daily smokers & & & & $80.9(238)$ \\
\hline \multicolumn{5}{|l|}{ Diabetes status, $\%$} \\
\hline Non-diabetic & $29.5(548)$ & $325(29.7)$ & $133(29.4)$ & $89(29.1)$ \\
\hline Prediabetic & $43.0(747)$ & $448(42.1)$ & $165(39.7)$ & $133(45.2)$ \\
\hline Diabetic & $27.5(523)$ & $296(28.2)$ & $145(30.9)$ & $82(25.7)$ \\
\hline Coronary heart disease, $\%$ & $6.9(108)$ & $5.4(54)$ & $9.9(33)$ & $7.2(21)$ \\
\hline Heart failure, \% & $3.6(49)$ & $2.5(26)$ & $5.7(13)$ & $4.4(10)$ \\
\hline ACE inhibitors, \% & $312(17.1)$ & $191(16.4)$ & 76 (18.9) & $45(16.8)$ \\
\hline Angiotensin receptor blockers, \% & $140(7.3)$ & $79(7.1)$ & $50(10.4)$ & $11(3.6)$ \\
\hline Beta blockers, \% & $201(12.1)$ & $114(10.9)$ & $68(18.2)$ & $19(6.9)$ \\
\hline
\end{tabular}

Data are presented as mean + SEM or \% (unweighted N) using weighted row percentages. It is HCHS/SOL publication policy to present weighted percentages. Unweighted percentages can be calculated based on unweighted N's listed. Chronic kidney disease defined by eGFR $<60 \mathrm{ml} / \mathrm{min}$.

BP, blood pressure; ECHO-SOL, Echocardiographic Study of Hispanics/Latinos; eGFR, estimated glomerular filtration rate; HCHS/SOL, Hispanic Community Health Study/Study of Latinos.

to atherosclerosis, the smoking status has been associated with structural changes in the heart including increasing left ventricle (LV) mass and abnormal LV geometry compared with non-smokers even after excluding those with major cardiovascular events such as interim atrial fibrillation and myocardial infarction. ${ }^{5}$ Research in the same cohort also found that current smoking status was associated with higher mean left atrium diameter. ${ }^{6}$

The relation of cigarette use with cardiac function has been less studied. Furthermore, while cigarette smoking or tobacco usage has generally been examined as a binary (yes/no) covariate, few major cohorts have assessed duration and intensity of cigarette use and their association with right and left ventricular cardiac structure and function via echocardiography. We hypothesised that younger age of smoking initiation (longer duration), increased number of cigarettes smoked per day (higher intensity) and total pack-years (an index of combined duration and intensity) would be associated with worse parameters of right-sided and left-sided cardiac structure and function.

\section{METHODS}

The Hispanic Community Health Study/Study of Latinos (HCHS/SOL) is a population-based longitudinal cohort study designed to examine multiple aspects of chronic disease affecting the Hispanic/Latino population of the United States. Details of study design and sample selection have been published previously. ${ }^{78}$ Briefly, Hispanics/ Latinos $(\mathrm{n}=16415)$ aged $18-74$ years and residing in four US metropolitan areas (Bronx, NY; Chicago, IL; Miami, FL; and San Diego, CA) were recruited between 2008 and 2011. Ineligibility criteria for the HCHS/SOL included being on active military service, not currently living at home, planning to move from the area in the next 6 months, unable to complete the study in English or Spanish, or unable to attend the clinic examination.

ECHO-SOL (Echocardiographic Study of Hispanics/ Latinos) was designed to provide echocardiographic measures characterising cardiac structure and function in a representative baseline subsample of the HCHS/ SOL. The ECHO-SOL used a stratified sampling design to assure that ECHO-SOL represented the Hispanic subgroup distribution found in each of four HCHS/ 
SOL field centres. A detailed description of the design, rationale and methods has been described elsewhere. ${ }^{9}$

${ }^{10}$ Eligibility criteria for inclusion in ECHO-SOL were: aged 45 years or older and having completed the HCHS/ SOL baseline visit within 36 months or less from the date of enrolment. The Institutional Review Board at Wake Forest School of Medicine and at each field centre provided approval and oversight of all study materials and activities.

\section{Smoking assessment}

Study examinations included completion of standardised clinical measurements and questionnaires, conducted between 2008 and 2011 by a bilingual interviewer in either English or Spanish. Lifetime history of cigarette smoking was elicited by the question, 'Have you ever smoked at least 100 cigarettes in your entire life?'. Other smoking-related variables included number of cigarettes per day, age at smoking initiation and periods of smoking cessation. Self-reported information was used to define current daily smokers, and to define intermittent or some-days smokers as current smokers who did not report using cigarettes on a daily basis. Among current daily smokers, we estimated lifetime pack-years based on age of smoking initiation, periods of quitting and average lifetime cigarettes smoked per day. Reproducibility of these smoking variables has been previously reported. ${ }^{11}$

\section{Echocardiographic protocol}

Trained sonographers performed standard echocardiography examinations, including spectral, colour and tissue Doppler, M-mode, 2D imaging with harmonics, optimising depth and sector width to maintain a high frame rate. ${ }^{912}$ All measures were performed by experienced sonographers at each field imaging centre as per American Society of Echocardiography (ASE) recommendations. ${ }^{9} 13-15$

Echocardiographic-measured and derived variables were used as follows: left ventricular mass (LVM) was determined from LV chamber size and wall thickness as per ASE guidelines. Relative wall thickness (RWT) was defined as $2 *(\mathrm{LV}$ posterior wall thickness/LV internal diastolic diameter). Pulse-wave Doppler was performed with the sample volume placed at the level of the mitral leaflet tips to obtain peak early (E) diastolic transmitral inflow velocities; and tissue Doppler to acquire mitral early diastolic $\left(e^{\prime}\right)$ annular velocities (the average of septal and lateral annular velocities was used). ${ }^{914} \mathrm{Left}$ ventricular ejection fraction (LVEF) was derived from volumetric assessments to measure end-diastolic volume (EDV) and end-systolic volume (ESV) and calculated as follows: $\mathrm{LVEF}=(\mathrm{EDV}-\mathrm{ESV}) / \mathrm{EDV} .^{15}$

Right ventricular (RV) end-diastolic area (RVAd) was measured via an RV-focused apical four-chamber view. Right ventricular fractional area change (RVFAC), tricuspid annular plane systolic excursion (TAPSE), right ventricular stroke volume (RVSV) and RV peak systolic (RV peak S) tissue Doppler velocity capture
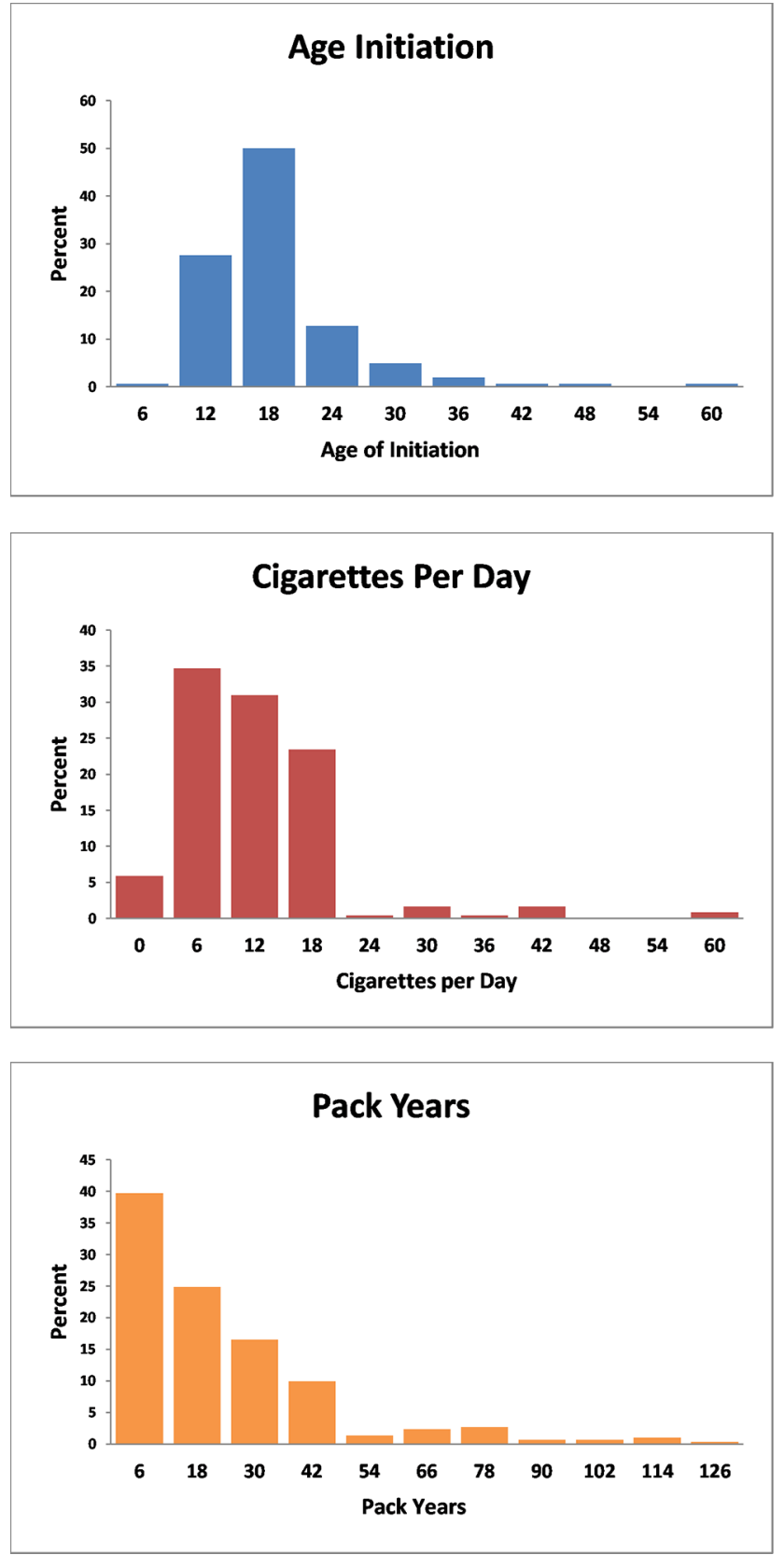

Figure 1 Histograms among current smokers.

different dimensions of RV systolic function. RVFAC is defined as [( $\mathrm{RV}$ end-diastolic area - RV end-systolic area) $/$ RV end-diastolic area $\times 100]$. RVFAC was obtained by tracing the RV endocardium from the annulus, along the free wall to the apex, back to the apex, then along the interventricular septum. TAPSE was measured by placing an M-mode cursor through the tricuspid annulus and measuring longitudinal annulus motion. RVSV was assessed using right ventricular outflow tract (RVOT) dimensions and pulse-wave Doppler for RVOT velocity time integral (VTI). TR max (maximum) velocity was measured using continuous wave Doppler through the tricuspid valve as an assessment of RV systolic pressures. ${ }^{16}$ Inter-reader reliability studies have been described and intraclass correlations were $>0.80$ for all measures. ${ }^{9}$ 


\section{Clinical characteristics}

The HCHS/SOL examination protocol has been previously published. ${ }^{17} 18$ Trained and certified clinic staff obtained all demographic characteristics and medical history using interviewer-administered questionnaires, as well as blood samples and clinical measurements, on all HCHS/SOL participants. Medication use was assessed by participants bringing all medications and supplements used during the last 4 weeks. These were reviewed and coded by clinic staff. Diabetes was defined as $^{1}$ : fasting serum glucose $>126 \mathrm{mg} / \mathrm{dL},{ }^{2}$ oral glucose tolerance test $>200 \mathrm{mg} / \mathrm{dL},{ }^{3}$ self-reported diabetes, ${ }^{4}$ glycosylated haemoglobin $>6.5 \%$, or ${ }^{5}$ taking antidiabetes medication or insulin. Height was measured to the nearest $0.1 \mathrm{~cm}$ and weight was measured to the nearest $0.1 \mathrm{~kg}$ with the use of a balance scale. Body mass index (BMI) was calculated as weight in kilograms divided by height in metres squared. After a $5 \mathrm{~min}$ rest, blood pressure was measured three times at $1 \mathrm{~min}$ intervals using an automated oscillometric device with the participant seated, using the average of the second and third blood pressure measurements. Hypertension was defined as a systolic blood pressure of $\geq 140 \mathrm{~mm} \mathrm{Hg}$, diastolic blood pressure of $\geq 90 \mathrm{~mm} \mathrm{Hg}$ or on antihypertensive treatment. Coronary heart disease was defined as history of myocardial infarction or revascularisation as well as ECG evidence of old myocardial infarction. The prevalence of heart failure was assessed based on self-reported history of physician-diagnosed clinical heart failure. Chronic kidney disease was defined by an estimated glomerular filtration rate (eGFR) $<60 \mathrm{ml} / \mathrm{min}$ per $1.73 \mathrm{~m}^{2}$; GFR was estimated from serum creatinine. ${ }^{19}$ Spirometry was conducted as previously described. ${ }^{20}$ Participants with prebronchodilator forced expiratory volume/forced vital capacity ratios less than 0.70 or less than the lower limit of normal were defined as having airflow obstruction.

\section{Statistical analysis}

Age, BMI, fasting glucose, heart rate, systolic and diastolic blood pressures, and total cholesterol are presented as continuous measures $\pm \mathrm{SE}$ due to the survey weighting. Other variables were presented as proportional frequencies \pm SEM. Mean measurements and SE of echocardiographic parameters according to smoking status were calculated using survey means. The association of smoking status (current vs never and former vs

Table 2 Mean values of echocardiographic parameters according to smoking status

\begin{tabular}{|c|c|c|c|c|c|c|c|c|c|c|c|}
\hline \multirow[b]{2}{*}{ Variable } & \multicolumn{3}{|c|}{ Never smokers } & \multicolumn{3}{|c|}{ Former smokers } & \multicolumn{4}{|c|}{ Current smokers } & \multirow[b]{2}{*}{$\begin{array}{l}\text { p (to } \\
\text { never } \\
\text { smokers) }\end{array}$} \\
\hline & Mean & Range & SEM & Mean & Range & SEM & $\begin{array}{l}p \text { (to } \\
\text { never } \\
\text { smokers) }\end{array}$ & Mean & Range & SEM & \\
\hline \multicolumn{12}{|c|}{ Left ventricular structure and function } \\
\hline LVEDV, mL & 81.9 & $36.0-168.0$ & 0.4 & 85.0 & $31.2-200.7$ & 1.5 & 0.09 & 86.6 & $34.6-184.1$ & 1.5 & 0.009 \\
\hline LVESV, mL & 32.6 & $8.4-90.3$ & 1.0 & 34.7 & $13.4-88.5$ & 0.7 & 0.02 & 36.4 & $12.9-137.1$ & 1.0 & 0.001 \\
\hline LVM, g & 149.7 & $63.6-469.6$ & 2.3 & 159.8 & 67.4-336.1 & 2.9 & 0.01 & 156.8 & $60.3-325.6$ & 2.9 & 0.09 \\
\hline RWT & 0.4 & $0.2-0.8$ & 0.01 & 0.4 & $0.2-0.8$ & 0.01 & 0.34 & 0.4 & $0.2-0.7$ & 0.01 & 0.11 \\
\hline$E / e^{\prime}$ & 10.1 & $4.3-28.0$ & 10.1 & 9.9 & $4.7-27.7$ & 0.2 & 0.38 & 9.5 & $4.5-28.0$ & 0.5 & 0.008 \\
\hline \multicolumn{12}{|c|}{ Right ventricular structure and function } \\
\hline $\begin{array}{l}\text { RV peak S } \\
\text { velocity }\end{array}$ & 12.0 & $5.3-25.8$ & 0.1 & 12.0 & $5.5-22.3$ & 0.14 & 0.92 & 11.4 & $3.6-20.2$ & 0.2 & 0.009 \\
\hline RVOT VTI, cm & 17.8 & $8.7-30.6$ & 0.2 & 17.0 & $9.5-31.3$ & 0.2 & 0.005 & 16.7 & $6.8-27.7$ & 0.2 & 0.0002 \\
\hline RVSV, mL & 49.3 & $13.9-141.4$ & 0.9 & 48.3 & $14.0-117.4$ & 1.3 & 0.51 & 45.7 & $10.4-120.5$ & 1.3 & 0.02 \\
\hline $\begin{array}{l}\text { TR max } \\
\text { peak gradient, } \\
\mathrm{mm} \mathrm{Hg}\end{array}$ & 18.3 & $0.1-3.3$ & 0.02 & 18.1 & $1.6-42.5$ & 0.5 & 0.67 & 16.2 & $1.5-44.8$ & 0.5 & 0.0003 \\
\hline $\begin{array}{l}\text { TR max } \\
\text { velocity, cm/s }\end{array}$ & 209.1 & $11.1-329.2$ & 2.0 & 207.5 & $63.3-325.8$ & 3.1 & 0.63 & 195.7 & $60.2-334.5$ & 3.2 & 0.0003 \\
\hline
\end{tabular}

Bold denotes statistically significant associations $(p \leq 0.01)$.

E/e', transmitral E velocity to mitral annulus e' velocity; LVEDV, left ventricular end-diastolic volume; LVEF, left ventricular ejection fraction; LVESV, left ventricular end-systolic volume; LVM, left ventricular mass; RVAd, right ventricular area at diastole; RVFAC, right ventricular fractional area change; RVOT, right ventricular outflow tract; RVSV, right ventricular stroke volume; RWT, relative wall thickness; TAPSE, tricuspid annular plane systolic excursion; TR, tricuspid regurgitation; VTI, velocity time integral. 
never) on echocardiographic measures was determined using survey linear regression. Nested multivariable linear regression models including sample weights based on survey statistics were used to describe the relationships between intensity and duration of tobacco usage (age at initiation of smoking, number of cigarettes per day on smoking days and pack-years) and echocardiographic indices of right and left ventricular structure and function. Multivariable models included age as a continuous variable with sex, BMI $<30 \mathrm{~kg} / \mathrm{m}^{2}$, hypertension status and diabetes status as categorical variables. Multivariable analysis was performed for RV structural and functional indices stratified by having airflow obstruction. Survey regression was used to determine significant differences between some-days smokers versus daily smokers with mean echocardiographic parameters. For
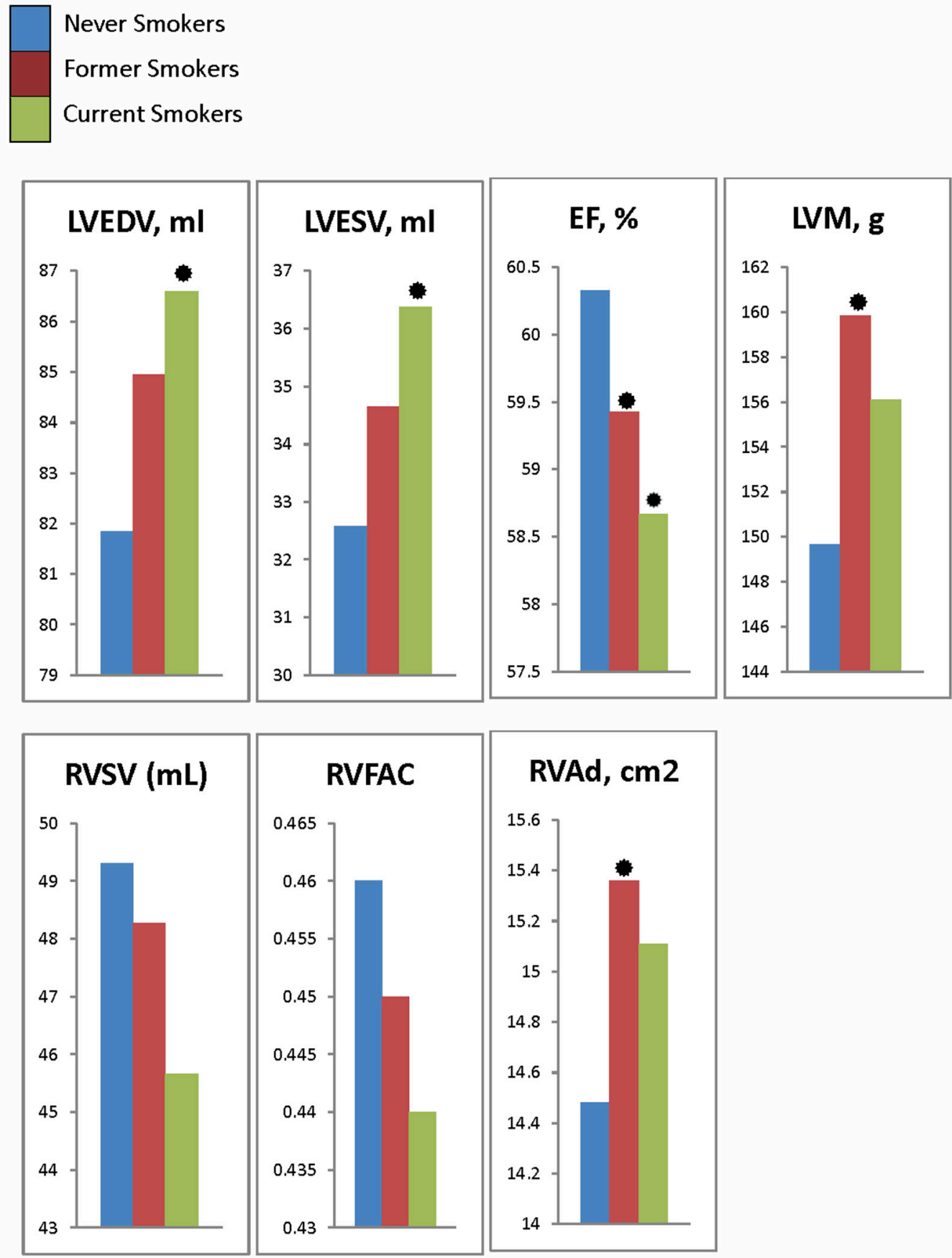

- Denotes statistically significant associations ( $p \leq 0.01)$; Reference is never smokers

Figure 2 Unadjusted means by smoking status. EF, ejection fraction; LVEDV, left ventricular end-diastolic volume; LVESV, left ventricular end-systolic volume; LVM, left ventricular mass; RVAd, right ventricular area at diastole; RVFAC, right ventricular fractional area change; RVSV, right ventricular stroke volume. 
current smokers, unweighted means were calculated for age of smoking initiation, number of cigarettes smoked per day and number of pack-years. Further, stratified analysis was performed based on three classifications: diabetes only $(n=27)$ excluding cases with hypertension, hypertension only $(n=82)$ excluding cases with diabetes and healthy cases $(n=96)$ excluding any diabetes, hypertension or obesity. All analyses were conducted using SAS V.9.4 (SAS Institute). SAS survey procedures and sampling weights were used to account for the complex survey design used in HCHS/SOL. A $p$ value $\leq 0.01$ was considered statistically significant.

\section{RESULTS}

ECHO-SOL included 1818 participants. Mean age of the ECHO-SOL target population in our analysis was 56.4 years and included $1187(57.4 \%)$ women. Mean BMI was 30.1. Only fewer than one-third (29.5\%) did not have prediabetes or diabetes and half were hypertensive. Over $90 \%$ were without chronic kidney disease. Fifty-five per cent reported never smoking, $25.7 \%$ identified as former smokers and $17.7 \%$ identified as current smokers. Former smokers were older, less likely to be female, more likely to be hypertensive and diabetic than current smokers. Almost one-quarter were being treated with renin-angiotensin system (RAS) blockade. RAS blockade and beta blockers use was more common among former smokers compared with never or current smokers (table 1). Among the 304 current smokers, the median age of initiation was 17.0 years with the middle $50 \%$ ranging from 14 to 20 years old and $\sim 5 \%$ who started smoking under the age of 12 (figure 1). The median number of pack years was 16.0 with the middle $50 \%$ ranging from 6.4 to 31.0. The median number of cigarettes smoked per day was 10.0 (also the most frequently reported value) with the middle $50 \%$ ranging from 5 to 17 cigarettes per day.

When compared with never smokers, former smokers were found to have significantly lower LVEF, RVOT VTI and LVM(table 2, figure 2). When compared with never smokers, current smokers had higher unadjusted mean LVEDV and LVESV, as well as significantly lower mean $\mathrm{RV}$ peak $\mathrm{S}$ velocity, TR max velocity, $\mathrm{E} / \mathrm{e}^{\prime}$ ratio and TR max pressure gradients. In linear regression models (table 3), both current and former smokers were found to have decreased RVOT VTI when compared with never smokers. Former smoking status was associated with decreased LVEF, increased LVM and increased RVAd, whereas current smoking status was associated with a lower RV peak S wave velocity, RVOT VTI, E/e' ratio, TR max pressure gradient and velocity. LVEDV and LVESV were increased in current smokers compared with never smokers.

Table 3 Regression coefficients for former or current smokers compared with never smokers

\begin{tabular}{|c|c|c|c|c|}
\hline \multirow[b]{2}{*}{ Variable } & \multicolumn{2}{|c|}{ Former versus never } & \multicolumn{2}{|c|}{ Current versus never } \\
\hline & $\begin{array}{l}\text { Regression } \\
\text { coefficient }\end{array}$ & $\mathbf{p}$ & $\begin{array}{l}\text { Regression } \\
\text { coefficient }\end{array}$ & $\mathbf{p}$ \\
\hline LVEDV, $\mathrm{mL}$ & 3.09 & 0.09 & 4.74 & 0.0088 \\
\hline LVESV, mL & 2.077 & 0.016 & 3.79 & 0.0008 \\
\hline RVAd, cm2 & 0.88 & 0.003 & 0.63 & 0.06 \\
\hline RWT & 0.007 & 0.34 & -0.011 & 0.11 \\
\hline$E / e^{\prime}$ & -0.21 & 0.38 & -0.67 & 0.008 \\
\hline \multicolumn{5}{|c|}{ Right ventricular structure and function } \\
\hline RVOT VTI, cm & -0.8 & 0.005 & -1.15 & 0.0002 \\
\hline RVSV, mL & -1.03 & 0.51 & -3.65 & 0.02 \\
\hline TR max peak gradient, $\mathrm{mm} \mathrm{Hg}$ & -0.22 & 0.67 & -2.06 & 0.0003 \\
\hline TR max velocity, cm/s & -1.57 & 0.63 & -13.4 & 0.0003 \\
\hline
\end{tabular}

Bold denotes statistically significant associations $(p \leq 0.01)$

$E / e^{\prime}$, transmitral E velocity to mitral annulus e' velocity; LVEDV, left ventricular end-diastolic volume; LVEF, left ventricular ejection fraction; LVESV, left ventricular end-systolic volume; LVM, left ventricular mass; RVAd, right ventricular area at diastole; RVFAC, right ventricular fractional area change; RVOT, right ventricular outflow tract; RVSV, right ventricular stroke volume; RWT, relative wall thickness; TAPSE, tricuspid annular plane systolic excursion; TR, tricuspid regurgutation; VTI, velocity time integral. 
Smoking Intensity and Duration is Associated with Cardiac Structure and Function
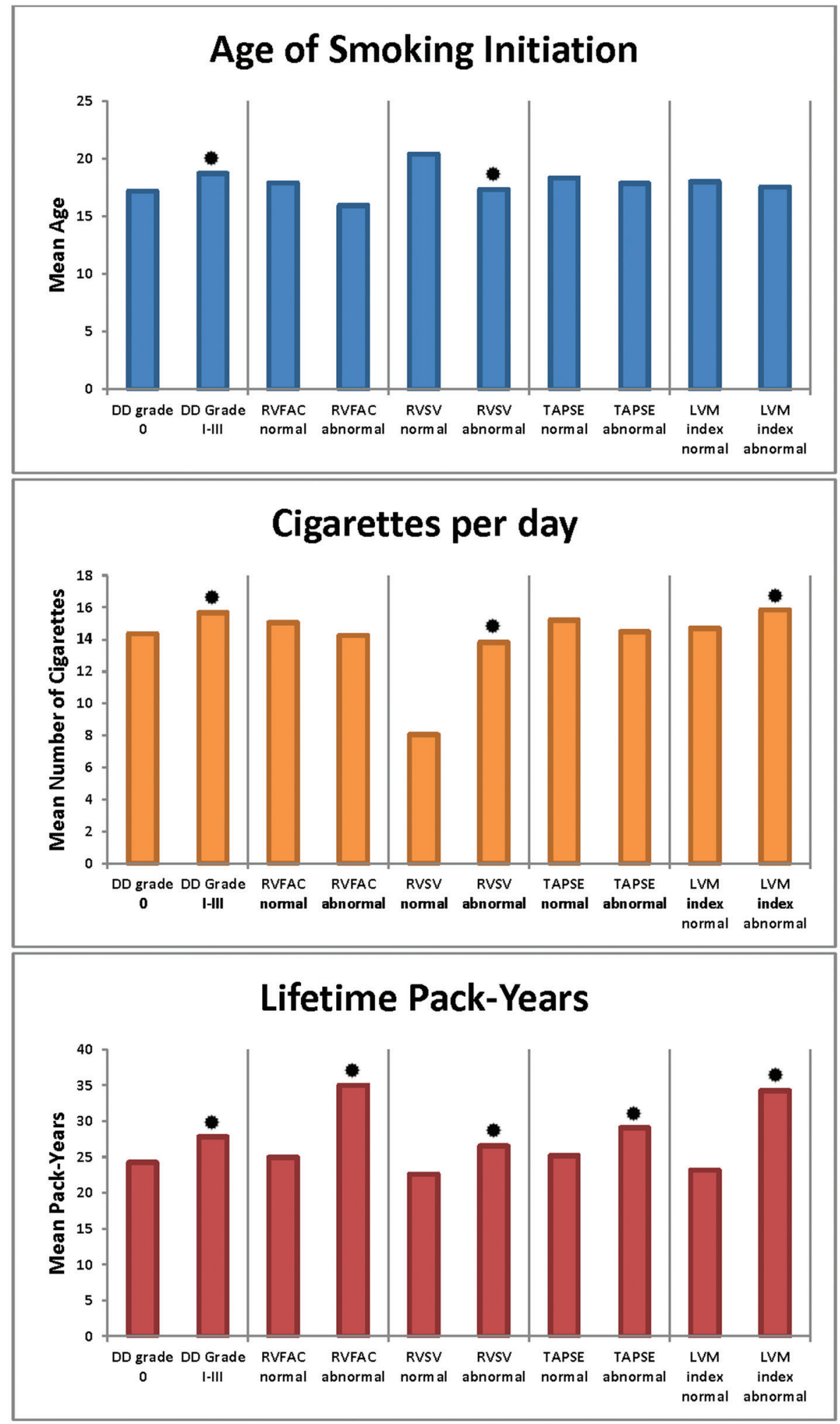

- Denotes statistically significant associations ( $\mathbf{p} \leq 0.01$ );

Cutoffs for abnormal indices: RVFAC $<0.32 \%$; RVSV $<60 \mathrm{cc}$; TAPSE $<17 \mathrm{~mm}$; LVM $>165 \mathrm{~g}$; RWT $>0.42$

Figure 3 Unadjusted means according to normal versus abnormal cardiac variables among current smokers. DD, diastolic dysfunction; LVM, left ventricular mass; RVFAC, right ventricular fractional area change; RVSV, right ventricular stroke volume; RWT, relative wall thickness; TAPSE, tricuspid annular plane systolic excursion. 
Table 4 Multivariate analysis of different measures of tobacco usage

\begin{tabular}{|c|c|c|c|c|c|c|}
\hline \multirow[b]{2}{*}{ Variable } & \multicolumn{2}{|c|}{ Age at initiation } & \multicolumn{2}{|c|}{ Cigarettes per day } & \multicolumn{2}{|c|}{ Lifetime pack-years } \\
\hline & $\begin{array}{l}\text { Coefficient } \\
\text { estimate }\end{array}$ & p Value & $\begin{array}{l}\text { Coefficient } \\
\text { estimate }\end{array}$ & p Value & $\begin{array}{l}\text { Coefficient } \\
\text { estimate }\end{array}$ & p Value \\
\hline \multicolumn{7}{|c|}{ Left ventricular structure and function } \\
\hline LVEDV, mL & 0.15 & 0.03 & 0.03 & 0.46 & -0.02 & 0.16 \\
\hline LVESV, mL & 0.059 & 0.05 & 0.014 & 0.52 & -0.01 & 0.18 \\
\hline LVEF, \% & -0.002 & 0.87 & -0.004 & 0.80 & -0.002 & 0.51 \\
\hline LVM, g & -0.29 & 0.01 & 0.58 & $<0.0001$ & 0.33 & $<0.0001$ \\
\hline RVAd, $\mathrm{cm}^{2}$ & 0.02 & 0.24 & 0.002 & 0.85 & 0.0008 & 0.76 \\
\hline RWT & -0.00009 & 0.82 & 0.003 & $<0.0001$ & 0.0007 & $<0.0001$ \\
\hline $\mathrm{E} / \mathrm{e}^{\prime}$ & 0.03 & 0.001 & 0.046 & $<0.0001$ & 0.009 & 0.0006 \\
\hline \multicolumn{7}{|c|}{ Right ventricular structure and function } \\
\hline RVFAC & -0.0007 & 0.14 & -0.0003 & 0.12 & -0.0006 & $<0.0001$ \\
\hline TAPSE, cm & 0.00004 & 0.98 & -0.0005 & 0.68 & -0.0015 & $<0.0001$ \\
\hline RV peak S & -0.017 & 0.03 & 0.017 & 0.0001 & -0.007 & $<0.0001$ \\
\hline RVOT VTI, cm & 0.05 & 0.0002 & -0.025 & 0.0009 & -0.014 & $<0.0001$ \\
\hline RVSV, mL & 0.63 & $<0.0001$ & -0.28 & $<0.0001$ & -0.07 & $<0.0001$ \\
\hline TR max peak gradient, mm Hg & 0.035 & 0.06 & 0.098 & $<0.0001$ & -0.01 & 0.02 \\
\hline TR max velocity, cm/s & 0.36 & 0.005 & 0.68 & $<0.0001$ & -0.05 & 0.16 \\
\hline
\end{tabular}

Adjusted for age, sex, hypertension, diabetes and BMI.

Bold denotes statistically significant associations ( $p \leq 0.01$ ).

BMI, body mass index; E/e', transmitral E velocity to mitral annulus e' velocity; LVEDV, left ventricular end-diastolic volume; LVEF, left ventricular ejection fraction; LVESV, left ventricular end-systolic volume; LVM, left ventricular mass; RVAd, right ventricular area at diastole; RVFAC, right ventricular fractional area change; RVOT, right ventricular outflow tract; RVSV, right ventricular stroke volume; RWT, relative wal thickness; TAPSE, tricuspid annular plane systolic excursion; TR, tricuspid regurgitation; VTI, velocity time integral.

Among current smokers, significant differences were seen for the mean ages of initiation among those with normal versus abnormal diastolic function and RVSV; for the mean number of cigarettes per day between those with normal versus abnormal diastolic function, RVSV and LVM; and for the mean number of pack-years among those with normal versus abnormal diastolic function, RVFAC, RVSV, TAPSE and LVM (figure 3). In multivariable-adjusted models (age, sex, obesity status, hypertension status and diabetes status) (table 4), among current smokers, increased duration of smoking as measured by lower age of smoking initiation was significantly associated with higher LVM. Lower age of initiation was associated with lower RVSV, higher TR max velocity and lowerE/e' ratio. The number of cigarettes smoked per day was associated with a higher LVM, RWT, TR max velocity, E/ $\mathrm{e}^{\prime}$ ratio, TR max pressure gradient, and with a lower RVSV and RVOT VTI. Increasing lifetime packyears was associated with higher $\mathrm{LVM}, \mathrm{E} / \mathrm{e}^{\prime}$ ratio and RWT. Conversely, increasing lifetime pack-years was also associated with lower RVFAC, lower TAPSE, lower RV peak $\mathrm{S}$ and lower RVSV.

Among current smokers, $19.1 \%$ were some-days smokers. Unadjusted mean values of echocardiographic parameters were compared between current smokers who smoked daily and those who were non-daily smokers (table 5). Daily smokers were found to have lower LVEF and impaired indices of RV function (TAPSE, RV peak $\mathrm{S}$ velocity, RVOT VTI, TR max peak gradient) along with higher mean LVM and $\mathrm{E} / \mathrm{e}^{\prime}$ ratio compared with non-daily smokers. Among those with measured airway obstruction, defined by abnormal spirometry, increasing smoking intensity and duration (younger age of initiation, increasing cigarettes per day and pack-years) was associated with decreasing RVSV (table 6). Among those with no airflow obstruction, a significant negative association emerged for increasing cigarettes per day with lower RVAd, RVFAC and TAPSE. TR max velocity and pressure gradient increased with cigarettes per day in both strata. Increasing pack-years was associated with decreased RVFAC, RVOT VTI and RVSV in both strata.

When those with coronary heart disease or heart failure were excluded, all the relationships for LVM and RVSV remained the same across all measurements of cigarette use. Additionally, there was no change in the impact that age of initiation had on RVOT VTI, E/ $\mathrm{e}^{\prime}$ ratio and TR max velocity. Significant associations of younger age of initiation with decreasing LVEDV, LVESV and TR max pressure gradient, as well as increasing cigarettes per day with increasing LVESV emerged. The associations between cigarettes per day and RV peak S, and RVOT VTI, as well as pack-years with TAPSE lost statistical significance (data not shown). Stratified analysis showed consistent and diffuse alterations of RV and LV structure 
Table 5 Means of current smokers (daily smokers versus non-daily smokers)

Non-daily smokers Daily smokers

\begin{tabular}{|c|c|c|c|c|c|}
\hline \multirow[b]{2}{*}{ Variable } & & & & & \\
\hline & Mean & SE & Mean & SE & $\begin{array}{l}\text { p (compared } \\
\text { with non- } \\
\text { daily) }\end{array}$ \\
\hline \multicolumn{6}{|l|}{ Left ventricular structure and function } \\
\hline LVEDV, $\mathrm{mL}$ & 85.5 & 3.6 & 86.9 & 1.6 & 0.43 \\
\hline LVESV, mL & 35.1 & 1.9 & 36.7 & 1.1 & 0.08 \\
\hline LVEF, \% & 60 & 1.0 & 58.4 & 0.7 & $<0.0001$ \\
\hline LVM, g & 148.1 & 4.0 & 158.0 & 3.4 & $<0.0001$ \\
\hline RWT & 0.4 & 0.01 & 0.39 & 0.01 & 0.017 \\
\hline$E / e^{\prime}$ & 8.8 & 0.3 & 9.6 & 0.3 & $<0.0001$ \\
\hline \multicolumn{6}{|l|}{ Right ventricular structure and function } \\
\hline RVAd, $\mathrm{cm}^{2}$ & 14.9 & 0.6 & 15.2 & 0.3 & 0.3 \\
\hline RVFAC & 0.45 & 0.01 & 0.44 & 0.01 & 0.07 \\
\hline TAPSE, cm & 2.2 & 0.05 & 2.01 & 0.04 & $<0.0001$ \\
\hline $\mathrm{RV}$ peak $\mathrm{S}$ velocity, $\mathrm{cm} / \mathrm{s}$ & 12.2 & 0.4 & 11.2 & 0.2 & $<0.0001$ \\
\hline RVOT VTI, cm & 17.5 & 0.4 & 16.5 & 0.3 & $<0.0001$ \\
\hline Right ventricular stroke volume, $\mathrm{mL}$ & 46.6 & 2.2 & 45.5 & 1.5 & 0.4 \\
\hline TR max peak gradient, $\mathrm{mm} \mathrm{Hg}$ & 17.3 & 1.1 & 15.9 & 0.6 & 0.0017 \\
\hline TR max velocity, $\mathrm{cm} / \mathrm{s}$ & 201.9 & 7.6 & 194.2 & 3.8 & 0.01 \\
\hline
\end{tabular}

Bold denotes statistically significant associations $(p \leq 0.01)$.

E/e', transmitral E velocity to mitral annulus e' velocity; LVEDV, left ventricular end-diastolic volume; LVEF, left ventricular ejection fraction; LVESV, left ventricular end-systolic volume; LVM, left ventricular mass; RVAd, right ventricular area at diastole; RVFAC, right ventricular fractional area change; RVOT, right ventricular outflow tract; RWT, relative wall thickness; TAPSE, tricuspid annular plane systolic excursion; Tr, tricuspid regurgitation; VTI, velocity time integral.

and function with different measures of tobacco usage most pronounced among diabetics and less so among those only hypertensive, or among only the healthy (see online supplemental table).

\section{DISCUSSION}

There were a number of observed dose-response relationships between intensity and duration of cigarette smoking with measures of right and left cardiac structure and function. Among current smokers, increased duration of smoking (as measured by younger age of initiation) was associated with worse measures of $\mathrm{LV}$ structure (increasing mass and RWT), worse LV diastolic function and worse measures of RV function (RVSV, RVFAC). Increasing number of cigarettes smoked per day was associated with increasing LVM, worse diastolic function, worse LV geometry and worse RV function. Lastly, increasing lifetime pack-years was associated with increasing LVM, worse LV geometry, worse diastolic function, more RV dilation and worse RV function.

Tobacco smoke is a complicated mixture of over 5000 compounds. ${ }^{21}$ Cigarette smoking can exert adverse cardiac effects through a number of different pathways. ${ }^{22}$ Although the relationship between smoking and atherosclerotic cardiovascular disease has been well demonstrated, the exact mechanisms through which smoking acts are myriad and incompletely defined..$^{23}$ Proposed mechanisms for adverse cardiovascular outcomes include increased sympathetic stimulation via nicotine, carbon monoxide exposure, endothelial dysfunction, direct cellular toxicity and oxidative stress which accelerates atherosclerosis and has prothrombotic effects. ${ }^{23}$ In animal models, tobacco smoke has been shown to induce ventricular hypertrophy and remodelling with associated increases in serum norepinephrine levels, nicotinamide adenine dinucleotide phosphate oxidase activity and mitogen-activated protein kinase activation. ${ }^{24} 25$ This suggests that smoking also causes cardiac damage at the microcellular level independent of atherosclerotic and thrombotic events. Smoking has a known association with increased arterial stiffness. ${ }^{26}$ The active ingredient in the chemical addiction to cigarettes, nicotine, directly stimulates the autonomic nervous system and increases sympathetic outflow. ${ }^{27-29}$ Acute exposure to nicotine has been found to increase heart rate, myocardial contractility and blood pressure. ${ }^{30}$ By inducing a chronic state of sympathetic activity, usage of cigarettes has the potential to cause chronic micro-increases in blood pressure; arterial stiffness and heart rate can induce cardiac remodelling, increase LVM and potentially lead to cardiomyopathy. With cardiac hypertrophy, impaired diastolic function would be expected. Further, 
Table 6 Multivariate analysis of right ventricular structure and function stratified by obstructive lung disease status

\begin{tabular}{|c|c|c|c|c|c|c|}
\hline \multirow[b]{2}{*}{ Variable } & \multicolumn{2}{|c|}{ Age of initiation } & \multicolumn{2}{|c|}{ Cigarettes per day } & \multicolumn{2}{|c|}{ Pack-years } \\
\hline & Estimate & $\mathbf{p}$ & Estimate & $\mathbf{p}$ & Estimate & $\mathbf{p}$ \\
\hline \multicolumn{7}{|l|}{ No airflow obstruction } \\
\hline RVAd, $\mathrm{cm}^{2}$ & 0.011 & 0.54 & 0.032 & 0.0191 & 0.001 & 0.87 \\
\hline RVFAC & -0.002 & 0.0008 & 0.0002 & 0.50 & -0.0004 & $<0.0001$ \\
\hline TAPSE, cm & -0.001 & 0.60 & 0.005 & 0.0089 & -0.0003 & 0.46 \\
\hline RV peak S velocity, $\mathrm{cm} / \mathrm{s}$ & -0.032 & 0.0004 & 0.029 & $<0.0001$ & $-4.3 \mathrm{E}-05$ & 0.98 \\
\hline RVOT VTI, cm & 0.070 & $<0.0001$ & -0.007 & 0.0137 & -0.011 & 0.0012 \\
\hline Right ventricular stroke volume, $\mathrm{mL}$ & 0.716 & $<0.0001$ & -0.193 & 0.0152 & -0.086 & 0.0001 \\
\hline TR max velocity, $\mathrm{cm} / \mathrm{s}$ & 0.003 & 0.0021 & 0.005 & 0.0049 & -0.0003 & 0.62 \\
\hline TR $\max P G, m m ~ H g$ & 0.020 & 0.19 & 0.065 & 0.0065 & -0.004 & 0.66 \\
\hline \multicolumn{7}{|l|}{ Airflow obstruction } \\
\hline RVAd, $\mathrm{cm}^{2}$ & 0.069 & 0.11 & -0.063 & $<0.0001$ & 0.010 & 0.07 \\
\hline RVFAC & 0.006 & 0.0003 & -0.001 & 0.0019 & -0.001 & $<0.0001$ \\
\hline TAPSE, cm & 0.019 & $<0.0001$ & -0.010 & $<0.0001$ & -0.003 & $<0.0001$ \\
\hline $\mathrm{RV}$ peak $\mathrm{S}$ velocity, $\mathrm{cm} / \mathrm{s}$ & 0.023 & 0.29 & -0.004 & 0.5073 & -0.009 & $<0.0001$ \\
\hline RVOT VTI, cm & 0.082 & 0.024 & -0.070 & $<0.0001$ & -0.035 & $<0.0001$ \\
\hline Right ventricular stroke volume, $\mathrm{mL}$ & 1.269 & 0.001 & -0.456 & $<0.0001$ & -0.144 & $<0.0001$ \\
\hline TR max velocity, $\mathrm{cm} / \mathrm{s}$ & 0.023 & 0.0005 & 0.006 & $<0.0001$ & 0.002 & $<0.0001$ \\
\hline TR max PG, mm Hg & 0.390 & 0.001 & 0.091 & $<0.0001$ & 0.029 & 0.0003 \\
\hline
\end{tabular}

Bold denotes statistically significant associations ( $p \leq 0.01)$.

$P G$, peak gradient; RVAd, right ventricular area at diastole; RVFAC, right ventricular fractional area change; RVOT, right ventricular outflow tract; TAPSE, tricuspid annular plane systolic excursion; TR, tricuspid regurgitation; VTI, velocity time integral.

smoking-related micro-alterations resulting in higher heart rate and blood pressure would increase myocardial oxygen demand, making the heart more susceptible to microvascular ischaemic damage. Such a proposed model could explain why we observed abnormalities in $\mathrm{LV}$ mass, LV geometry and diastolic function related to increased cigarette smoke in the absence of known clinical coronary arterial disease.

While much of the epidemiologic research on the cardiovascular effects of smoking has focused on atherosclerotic disease, few work has examined cardiac functional aspects. The effects of nicotine on cardiac function have been studied using snus, a form of smokeless tobacco. Otherwise, healthy regular snus users considered free of cardiovascular disease had no significant change in systolic parameters but did have a prolonged deceleration times compared with non-nicotine users. ${ }^{31}$ Acute nicotine exposure was also found to cause a significant decrease and delay in relaxation of both right and left ventricles. ${ }^{32}$ Studies in healthy subjects have demonstrated acute impairment of LV diastolic function and inducement of a hyperdynamic RV in response to smoking a single cigarette. ${ }^{33}$ Smoking is also a risk factor for coronary spasm both in the presence and absence of atherosclerotic coronary artery disease. ${ }^{345}$ This may be due to the decreased nitric oxide generation which has been found in smokers with impaired endothelium-dependent vasodilation. ${ }^{36}$ Decreased nitric oxide activity has also been observed in the coronary arteries of smokers. ${ }^{37}$ All of these pathways combine to form an environment where the myocardium may be more susceptible to microvascular insults, leading to chronic damage and fibrosis affecting both cardiac structure and function. Many of these mechanisms have also been implicated in smoking as an inducer of chronic kidney disease. ${ }^{38}$ Our observed relationships may be the result of microvascular or inflammatory insults on the myocardium, sympathetic stimulation, coronary vasoconstriction, oxidative stress or toxic chemical exposure. Of course, the injury models described here would have effects on the LV and potentially on the RV.

The inclusion of comprehensive right heart echocardiography is important when studying the cardiac effects of tobacco. Prior studies of the cardiac alterations of smoking did not include assessment of the right heart or pulmonary function. Our study employed multiple measures of right heart function (RVFAC, RV peak S, RVSV and TAPSE) and found that altered RV function was associated with more cigarettes smoked and that this association was more consistent among those with impairment of lung function. The RV may be involved in whatever ischaemic or myopathic process is being imparted on the $\mathrm{LV}$, helping to explain our findings of impaired RV function with increasing cigarette usage. Cigarette smoking is also the most common risk factor for development of chronic obstructive pulmonary disease (COPD) ${ }^{39}$ COPD is associated with increased blood pressures in the pulmonary 
circuit $^{40}$ and RV enlargement, LV enlargement, as well as LV systolic and diastolic dysfunction. ${ }^{41}$ Even subclinical impairment of lung function has been shown to be associated with LV systolic and diastolic dysfunction ${ }^{42}$ which could potentially contribute to RV dysfunction as well.

Prior studies of the cardiac effects of tobacco were mostly racially homogenous and did not include Hispanics. To our knowledge, this is one of the first studies evaluating the association of exposure to smoking using measures of smoking intensity and duration (cigarettes per day, age of smoking initiation and lifetime pack-years of smoking) with comprehensive measures of cardiac structure and function including RV parameters and LV diastolic function in Hispanics/Latinos. Prior studies were mostly racially homogenous and did not include Hispanics/Latinos; they were 'piecemeal' in their assessment of cardiac structure and function whereas we provided a single comprehensive assessment. Further, compared with the report from the Atherosclerosis Risk in Communities study, ${ }^{43}$ our population is significantly younger by an average of 20 years with an average 16 lower pack-years of smoking but nevertheless showed the adverse cardiomyopathic effects of smoking intensity and duration. An important implication of our study is that cigarette smoking exposure may impact the development of cardiomyopathy in a direct, continuous fashion. The potential contribution of tobacco exposure to cardiomyopathy development and to the ongoing heart failure epidemic deserves further study and public health vigilance.

This study has some limitations. Our study was cross-sectional study and observational, hence we cannot exclude residual confounding, we cannot assess for temporality of the relations, and we cannot establish a causal relation between smoking and the echocardiographic measures. While it is advantageous to collect many structural and functional indices to paint a more complete picture, it carries the issue of multiple testing and type I error. To compensate for this, we set a more rigorous $\mathrm{p}$ value at 0.01 . Finally, there may be differential responses to chronic cigarette use based on race or ethnicity which may limit the generalisability of our results.

Our study provides evidence that there are cardiac abnormalities of structure and function associated with the intensity and duration of cigarette use in a relatively young cohort. Many of these relationships persisted even after excluding those with known coronary artery disease or heart failure. Our results imply that patients are likely damaging their hearts in a measurable fashion by smoking even if they have not suffered a clinical cardiovascular event. Descriptions of these cardiac abnormalities could serve as important data points for clinicians to use when counselling their patients on tobacco cessation. Traditionally, pack-years has been a standard clinical history assessment of total tobacco exposure. Our results are evidence that indices of both duration and intensity of usage are associated with abnormalities in multiple echocardiographic measures involving both the right and the left sides of the heart. From a research standpoint, more comprehensive assessments of tobacco or nicotine product usage should be used in future cohorts.

Author affiliations

${ }^{1}$ Wake Forest School of Medicine, Winston-Salem, North Carolina, USA

${ }^{2}$ Einstein College of Medicine, Bronx, North Carolina, USA

${ }^{3}$ University of Virginia Health System, Charlottesville, Virginia, USA

${ }^{4}$ University of Illinois at Chicago, Chicago, Illinois, USA

${ }^{5}$ San Diego State University, San Diego, California, USA

${ }^{6}$ University of North Carolina, Chapel Hill, North Carolina, USA

${ }^{7}$ Johns Hopkins Health System, Baltimore, Maryland, USA

${ }^{8}$ Boston University School of Medicine, Boston, Massachusetts, USA

${ }^{9}$ American Heart Association, Louisville, Kentucky, USA

${ }^{10}$ University of Louisville, Louisville, Kentucky, USA

Acknowledgements The authors acknowledge the investigators, the staff, and the participants of HCHS/SOL and ECHO-SOL for their dedication and commitment to the success of this study. Investigators website: http://www.cscc.unc.edu/hchs/.

Contributors All authors have read and approved the paper, have met the criteria for authorship as established by the International Committee of Medical Journals Editors, believe that the paper represents honest work, and are able to verify the validity of the results reported.

Funding The HCHS/SOL was carried out as a collaborative study supported by contracts from the National Heart, Lung, and Blood Institute (NHLBI) to the University of North Carolina (N01- HC65233), University of Miami (N01-HC65234), Albert Einstein College of Medicine (N01-HC65235), Northwestern University (N01-HC65236) and San Diego State University (N01-HC65237). The following institutes/centres/offices contribute to the HCHS/SOL through a transfer of funds to the NHLBI: National Institute on Minority Health and Health Disparities, National Institute on Deafness and Other Communication Disorders, National Institute of Dental and Craniofacial Research, National Institute of Diabetes and Digestive and Kidney Diseases, National Institute of Neurological Disorders and Stroke, NIH Institution-Office of Dietary Supplements. ECH0-SOL was supported by a grant from the NHLBI (R01 HL104199, Epidemiologic Determinants of Cardiac Structure and Function among Hispanics: Carlos J. Rodriguez, MD, MPH, Principal Investigator). An NHLBI T32HL076132 (to Adam Leigh, MD) also provided partial support for this manuscript. Research reported in this publication was also supported by the AHA Tobacco Regulation and Addiction Center (A-TRAC); grant number HL120163 from the NHLBI and the FDA Center for Tobacco Products. The content is solely the responsibility of the authors and does not necessarily represent the official views of the NIH or the FDA.

Competing interests None declared.

Ethics approval Institutional Review Board.

Provenance and peer review Not commissioned; internally peer reviewed.

Data sharing statement All HCHS-SOL data will be included in a limited access database that will be available to the larger scientific public as part of the main HCHS-SOL study database for public use. It will be encouraged that all use of data and publications from ancillary studies invite the participation of the ancillary study $\mathrm{Pl}$ and co-investigators.

Open Access This is an Open Access article distributed in accordance with the Creative Commons Attribution Non Commercial (CC BY-NC 4.0) license, which permits others to distribute, remix, adapt, build upon this work non-commercially, and license their derivative works on different terms, provided the original work is properly cited and the use is non-commercial. See: http://creativecommons.org/ licenses/by-nc/4.0/

Correction notice This paper has been amended since it was published Online First. Owing to a scripting error, some of the publisher names in the references were replaced with 'BMJ Publishing Group'. This only affected the full text version, not the PDF. We have since corrected these errors and the correct publishers have been inserted into the references.

(c) Article author(s) (or their employer(s) unless otherwise stated in the text of the article) 2017. All rights reserved. No commercial use is permitted unless otherwise expressly granted.

\section{REFERENCES}

1. Centers for Disease Control and Prevention, National Center for Chronic Disease Prevention and Health Promotion, Office on Smoking 
and Health. How tobacco smoke causes disease: the Biology and Behavioral Basis for Smoking-Attributable Disease: a Report of the Surgeon General. Publications and reports of the Surgeon General. Atlanta, GA: Centers for Disease Control and Prevention (US), 2010.

2. Lloyd-Jones DM, Hong Y, Labarthe D, et al. Defining and setting national goals for cardiovascular health promotion and disease reduction: the american Heart Association's strategic Impact Goal through 2020 and beyond. Circulation 2010;121:586-613.

3. Centers for Disease Control and Prevention, Office on Smoking. smoking and tobacco use. Fact Sheet; Adult Cigarette Smoking in the United States. http://www.cdc.gov/tobacco/data_statistics/fact sheets/adult data/cig_smoking/ (accessed 2901 2016).

4. Yancy CW, Jessup M, Bozkurt B, et al. 2013 ACCF/AHA guideline for the management of Heart failure. A Report of the American College of Cardiology Foundation/American Heart Association Task Force on Practice guidelines. J Am Coll Cardiol 2013;135.

5. Gidding SS, Liu K, Colangelo LA, et al. Longitudinal determinants of left ventricular mass and geometry: the Coronary Artery Risk Development in Young Adults (CARDIA) Study. Circ Cardiovasc Imaging 2013;6:769-75.

6. Gidding SS, Carnethon MR, Daniels S, et al. Low cardiovascular risk is associated with favorable left ventricular mass, left ventricular relative wall thickness, and left atrial size: the CARDIA study. J Am Soc Echocardiogr 2010;23:816-22.

7. Lavange LM, Kalsbeek WD, Sorlie PD, et al. Sample design and cohort selection in the Hispanic Community Health Study/Study of Latinos. Ann Epidemiol 2010;20:642-9.

8. Sorlie PD, Avilés-Santa LM, Wassertheil-Smoller S, et al. Design and implementation of the Hispanic Community Health Study/Study of Latinos. Ann Epidemiol 2010;20:629-41.

9. Rodriguez CJ, Dharod A, Allison MA, et al. Rationale and Design of the Echocardiographic Study of Hispanics/Latinos (ECHO-SOL). Ethn Dis 2015;25:180-6.

10. Mehta H, Armstrong A, Swett K, et al. Burden of Systolic and Diastolic Left Ventricular Dysfunction Among Hispanics in the United States: Insights From the Echocardiographic Study of Latinos. Circ Heart Fail 2016;9:e002733.

11. Kaplan RC, Bangdiwala SI, Barnhart JM, et al. Smoking among U.S. Hispanic/Latino adults: the Hispanic community health study/study of Latinos. Am J Prev Med 2014;46:496-506.

12. Mehta $\mathrm{H}$, Armstrong $\mathrm{A}$, Swett $\mathrm{K}$, et al. Burden of Systolic and Diastolic Left Ventricular Dysfunction Among Hispanics in the United States: Insights From the Echocardiographic Study of Latinos. Circ Heart Fail 2016;9:e002733.

13. Lang RM, Bierig M, Devereux RB, et al; Chamber Quantification Writing GroupAmerican Society of Echocardiography's Guidelines and Standards CommitteeEuropean Association of Echocardiography. Recommendations for chamber quantification: a report from the American Society of Echocardiography's Guidelines and Standards Committee and the Chamber Quantification Writing Group, developed in conjunction with the European Association of Echocardiography, a branch of the European Society of Cardiology. J Am Soc Echocardiogr 2005;18:1440-63.

14. Nagueh SF, Smiseth OA, Appleton CP, et al. Recommendations for the Evaluation of Left Ventricular Diastolic Function by Echocardiography: An Update from the American Society of Echocardiography and the European Association of Cardiovascular Imaging. J Am Soc Echocardiogr 2016;29:277-314.

15. Lang RM, Badano LP, Mor-Avi V, et al. Recommendations for Cardiac Chamber Quantification by Echocardiography in Adults: An Update from the American Society of Echocardiography and the European Association of Cardiovascular Imaging. Journal of the American Society of Echocardiography 2015;28:1-39.

16. Rudski LG, Lai WW, Afilalo J, et al. Guidelines for the echocardiographic assessment of the right heart in adults: a report from the American Society of Echocardiography endorsed by the European Association of Echocardiography, a registered branch of the European Society of Cardiology, and the Canadian Society of Echocardiography. J Am Soc Echocardiogr 2010;23:685-713.

17. Daviglus ML, Talavera GA, Avilés-Santa ML, et al. Prevalence of major cardiovascular risk factors and cardiovascular diseases among Hispanic/Latino individuals of diverse backgrounds in the United States. JAMA 2012;308:1775-84

18. Rodriguez CJ, Cai J, Swett K, et al. High Cholesterol Awareness, Treatment, and Control Among Hispanic/Latinos: Results From the Hispanic Community Health Study/Study of Latinos. J Am Heart Assoc 2015;4:e001867.
19. Ricardo AC, Flessner MF, Eckfeldt JH, et al. Prevalence and Correlates of CKD in Hispanics/Latinos in the United States. Clin J Am Soc Nephrol 2015;10:1757-66

20. Barr RG, Avilés-Santa L, Davis SM, et al. Pulmonary Disease and Age at Immigration among Hispanics. Results from the Hispanic Community Health Study/Study of Latinos. Am J Respir Crit Care Med 2016;193:386-95.

21. Borgerding $\mathrm{M}, \mathrm{Klus} \mathrm{H}$. Analysis of complex mixtures--cigarette smoke. Exp Toxicol Pathol 2005;57 (Suppl 1):43-73.

22. Salahuddin S, Prabhakaran D, Roy A. Pathophysiological Mechanisms of Tobacco-Related CVD. Glob Heart 2012;7:113-20.

23. Ambrose JA, Barua RS. The pathophysiology of cigarette smoking and cardiovascular disease: an update. J Am Coll Cardiol 2004;43:1731-7.

24. Rafacho BP, Azevedo PS, Polegato BF, et al. Tobacco smoke induces ventricular remodeling associated with an increase in NADPH oxidase activity. Cell Physiol Biochem 2011;27:305-12.

25. Gu L, Pandey V, Geenen DL, et al. Cigarette smoke-induced left ventricular remodelling is associated with activation of mitogenactivated protein kinases. Eur J Heart Fail 2008;10:1057-64.

26. Markus MR, Stritzke J, Baumeister SE, et al; MONICA/KORA Augsburg Cohort Study. Effects of smoking on arterial distensibility, central aortic pressures and left ventricular mass. Int $J$ Cardiol 2013;168:2593-601.

27. Cryer PE, Haymond MW, Santiago JV, et al. Norepinephrine and epinephrine release and adrenergic mediation of smoking-associated hemodynamic and metabolic events. N Engl J Med 1976;295:573-7.

28. Middlekauff HR, Park J, Moheimani RS. Adverse effects of cigarette and noncigarette smoke exposure on the autonomic nervous system: mechanisms and implications for cardiovascular risk. J Am Coll Cardiol 2014;64:1740-50

29. Narkiewicz K, van de Borne PJ, Hausberg M, et al. Cigarette smoking increases sympathetic outflow in humans. Circulation 1998;98:528-34.

30. Haass M, Kübler W. Nicotine and sympathetic neurotransmission. Cardiovasc Drugs Ther 1997;10:657-65.

31. Sundström D, Waldenborg M, Emilsson K. Acute effects on the ventricular function in Swedish snuffers: an echocardiographic study. Clin Physiol Funct Imaging 2012;32:106-13.

32. Sundström D, Waldenborg M, Magnuson A, et al. Chronic nicotine effects on left ventricular function in healthy middle-aged people: an echocardiographic study. Clin Physiol Funct Imaging 2013;33:317-24.

33. Giacomin E, Palmerini E, Ballo P, et al. Acute effects of caffeine and cigarette smoking on ventricular long-axis function in healthy subjects. Cardiovasc Ultrasound 2008:6:9.

34. Sugiishi M, Takatsu F. Cigarette smoking is a major risk factor for coronary spasm. Circulation 1993;87:76-9.

35. Winniford MD, Wheelan KR, Kremers MS, et al. Smoking-induced coronary vasoconstriction in patients with atherosclerotic coronary artery disease: evidence for adrenergically mediated alterations in coronary artery tone. Circulation 1986;73:662-7.

36. Barua RS, Ambrose JA, Eales-Reynolds LJ, et al. Dysfunctional endothelial nitric oxide biosynthesis in healthy smokers with impaired endothelium-dependent vasodilatation. Circulation 2001;104:1905-10.

37. Kugiyama K, Yasue $\mathrm{H}$, Ohgushi M, et al. Deficiency in nitric oxide bioactivity in epicardial coronary arteries of cigarette smokers. J Am Coll Cardiol 1996;28:1161-7.

38. Orth SR, Hallan SI. Smoking: a risk factor for progression of chronic kidney disease and for cardiovascular morbidity and mortality in renal patients--absence of evidence or evidence of absence? Clin J Am Soc Nephrol 2008;3:226-36.

39. Løkke A, Lange P, Scharling $H$, et al. Developing COPD: a 25 year follow up study of the general population. Thorax 2006;61:935-9.

40. Simonneau G, Gatzoulis MA, Adatia I, et al. Updated clinical classification of pulmonary hypertension. J Am Coll Cardiol 2013;62:D34-D41.

41. Freixa X, Portillo K, Paré C, et al; PAC-COPD Study Investigators. Echocardiographic abnormalities in patients with COPD at their first hospital admission. Eur Respir J 2013;41:784-91.

42. Baum C, Ojeda FM, Wild PS, et al; Gutenberg Health Study investigators. Subclinical impairment of lung function is related to mild cardiac dysfunction and manifest heart failure in the general population. Int J Cardiol 2016;218:298-304.

43. Nadruz W, Claggett B, Gonçalves A, et al. Smoking and Cardiac Structure and Function in the Elderly: The ARIC Study (Atherosclerosis Risk in Communities). Circ Cardiovasc Imaging 2016;9:e004950. 